\title{
Surface Effects on the Diffraction of P Wave by an Arbitrary Shaped Cavity
}

\author{
Zhiying Ou*, Hongjun Han \\ School of Science, Lanzhou University of Technology, Gansu, Lanzhou, China \\ Email: *zhiyingou@163.com
}

How to cite this paper: Ou, Z.Y. and Han, H.J. (2019) Surface Effects on the Diffraction of $\mathrm{P}$ Wave by an Arbitrary Shaped Cavity. Open Journal of Applied Sciences, 9, 439-450.

https://doi.org/10.4236/ojapps.2019.96036

Received: May 21, 2019

Accepted: May 31, 2019

Published: June 3, 2019

Copyright $\odot 2019$ by author(s) and Scientific Research Publishing Inc. This work is licensed under the Creative Commons Attribution International License (CC BY 4.0).

http://creativecommons.org/licenses/by/4.0/

\section{(c) (i) Open Access}

\begin{abstract}
This paper presents the effects of surface effects in the cavity of variable curvature. The wave function expansion method and the conformal mapping method are used in the solution of dynamic stress concentration factor around an irregularly shaped cavity at nano-scale. The stress boundary conditions on the surface are obtained by using the generalized Young-Laplace equation. The results show that the degree of stress concentration becomes more obvious with curvature increasing. Taking the elliptical cavity as an example, the influence of the ration of the major and minor axis of the ellipse, the numbers of the incident wave and the surface effects on the dynamic stress concentration factor are analyzed. The ration of the major and minor axis, the incident wave frequency and the surface effects show the pronounced effects on the dynamic stress concentration distributions.
\end{abstract}

\section{Keywords}

Conformal Mapping, Dynamic Stress Concentration Factor, Irregularly Shaped Cavity, Surface Effect

\section{Introduction}

Nano-structured materials and nano-sized structural elements have unique mechanical and physical properties. Atoms near the surface and interface of solids have different equilibrium positions and energies from that of the bulk material. Therefore, the surface and interface of solids display different mechanical properties compared with their bulk parts. To account for the effects of surface or interface, Gurtin et al. established the surface elasticity theory, which a surface is regarded as a negligibly thin membrane adhered to the bulk without slipping. The presence of surface stress gives rise to a non-classical boundary condition, which shows a good agreement with directly atomic simulations [1] [2]. There- 
fore, at the micro- and nano-sized, the surface elasticity theory has been adopted to elucidate many phenomena. Dingreville et al. [3] addressed the surface effects on the size-dependent elastic behaviors of nano-sized structural elements. Although such size-dependency is negligible for conventional structural elements, it becomes significant when one of the dimensions of the element shrinks to nanometers. For composites containing nano-sized inhomogeneities, the accommodation of surface or interface energy predicted the size-dependent effective moduli [4] [5]. Using of surface elasticity theory, Lei et al. [6] studied the effects of surface stress on the contact problem at nanometers, in which the contact mechanical properties are remarkably different from those in bulk solids.

In fact, the surface elasticity theory can be applied not only to the statics analysis, but also to the dynamics analysis. In the framework of surface elasticity theory, the scattering of plane compressional and shear waves by a single nano-sized coated fiber and the multiple scattering by two cylinder inclusions, which embedded in an elastic matrix is studied by Ou and Lee using the method of eigenfunction expansion [7] [8]. Zhang et al. [9] used the wave function expansion method to study the effect of nano-sized arrays on the longitudinal wave diffraction in elastic media, and gave the corresponding elastic diffraction fields. Ru et al. [10] considered the SV wave multiple scattering caused by a cluster of nano-cylindrical holes. Using the displacement potential method and the wave function expansion method, they derived the scattering field around the hole. $\mathrm{Ou}$ et al. [11] studied the effects of semi-cylindrical inclusions on the scattering of plane $\mathrm{P}$ wave in an elastic half-plane. The results show that surface energy has a significant effect on the scattering of plane $\mathrm{P}$ wave as the radius of the semi-cylindrical inclusions shrink to nanometers. Wang et al. [12] [13] used Gurtin's surface elasticity model to analyze the diffraction of elastic wave by a nanosized inhomogeneity, and they also demonstrated that the surface energy has a significant effect on the diffraction of the elastic wave when the cavity radius is reduced to the nanoscale.

The above only consider the effects of surface effects in the case of constant curvature. However, in the preparation process of materials, cracks, inclusions, holes and other defects with variable curvature are often brought, such as elliptical and ellipsoidal cavities. Liu et al. [14] presented the solutions of two dimensional elastic wave equations in terms of complex wave functions and general expressions for boundary conditions for steady state incident waves. Dynamic stresses around a cavity of arbitrary shape are then expressed in a series of complex domain functions. The coefficient of the series can be determined by truncating a set of infinite algebraic equations. The values of dynamic stress concentration factor for circular and elliptical cavities as two examples are given in his paper. Ou et al. [15] studied stress concentration around a nanosized arbitrarily shaped hole in elastic half-space under uniformly distributed load. The maximum value of the hoop pressure is obtained near the maximum point of curvature. Based on the elastic waves diffraction theory [16] [17] [18], this paper 
mainly studies the diffraction of $\mathrm{P}$ wave by arbitrary shape cavity at the nano-scale, and gives a method for estimating the magnitude of the dynamic stress concentration factor (DSCF).

\section{Wave Function}

Assume a harmonically time-varying plane $\mathrm{P}$ wave propagating at an angle $\alpha$ with respect to the $x$-axis with circular frequencies $\omega$ as shown in Figure 1. The elastic medium is defined by the Lames constants $\lambda, \mu$ and the density $\rho$.

Such an incident wave can be described by a displacement potential in the $x-y$ plane as

$$
\phi^{\mathrm{inc}}(x, y)=\phi_{0} \exp \left[\mathrm{I} k_{\mathrm{p}}(x \cos (\alpha)+y \sin (\alpha))\right] .
$$

where $\phi_{0}$ is the amplitude of incident wave, the superscript inc represents the incident wave, $k_{p}=\omega / c_{p}$ is the wave number of $\mathrm{P}$ wave, $c_{p}=\sqrt{(\lambda+2 \mu) / \rho}$ is the wave velocity of $\mathrm{P}$ wave, $I=\sqrt{-1}$. For the convenience of calculation, the time-dependent term $\mathrm{e}^{-\mathrm{I} \omega t}$ is suppressed throughout.

Based on the complex function theory, we introduce complex variables $z=x+\mathrm{I} y$, Equation (1) is then transformed to the following form

$$
\phi^{\mathrm{inc}}(z, \bar{z})=\phi_{0} \exp \left[\frac{\mathrm{I} k_{p}}{2}\left(z \mathrm{e}^{-\mathrm{I} \alpha}+\bar{z} \mathrm{e}^{\mathrm{I} \alpha}\right)\right] .
$$

When the incident wave impinges upon the irregularly shaped cavity boundary, two waves are reflected from the boundary [19]. They are a P wave and an $\mathrm{S}$ wave, which their displacement potential functions can be expressed as follows, respectively

$$
\begin{aligned}
& \phi^{\mathrm{ref}}(z, \bar{z})=\sum_{n=-\infty}^{\infty} A_{n} H_{n}^{(1)}\left(k_{p}|z|\right)\left(\frac{z}{|z|}\right)^{n}, \\
& \psi^{\mathrm{ref}}(z, \bar{z})=\sum_{n=-\infty}^{\infty} B_{n} H_{n}^{(1)}\left(k_{s}|z|\right)\left(\frac{z}{|z|}\right)^{n} .
\end{aligned}
$$

where the superscript ref represents the reflect wave, $A_{n}$ and $B_{n}$ are arbitrary expansion coefficients to be determined. $k_{s}=\omega / c_{s}$ is the wave number of SV wave, $c_{s}=\sqrt{\mu / \rho}$ is the wave velocity of SV wave. $H_{n}^{(1)}(\cdot)$ is the nth Hankel function of the first kind. Hankel function is related to the Bessel functions of the first kind $J_{n}(\cdot)$ and the second kind $Y_{n}(\cdot)$ by $H_{n}^{(1)}(\cdot)=J_{n}(\cdot)+\mathrm{I} \cdot Y_{n}(\cdot)$.

\section{Solution of the Problem}

In this section, we use the variable $\eta=\zeta+\mathrm{I} \xi$. For wave scattering problems involving noncircular boundary [20] [21], it is possible to transform the domain $\mathrm{L}$ external to a closed curve in the $z$-plane into the domain $\mathrm{S}$ bounded by a unit circle in the $\eta$-plane by means of the conformal mapping in Figure 2.

The mapping function $\omega(\eta)$ of the following form [22] [23] 


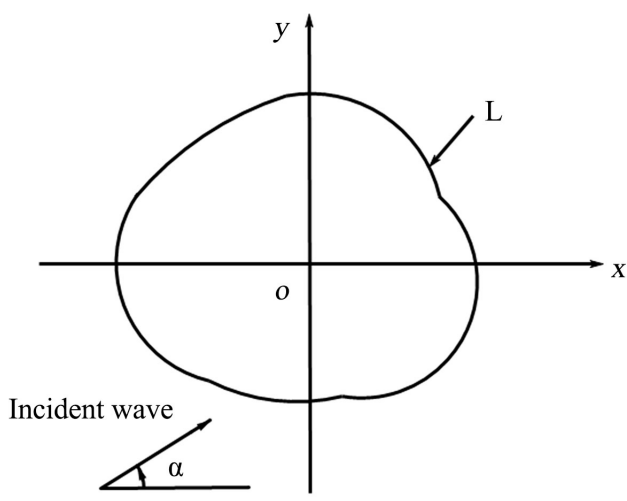

Figure 1. $\mathrm{P}$ wave propagating at an angle $\alpha$ with respect to the $x$-axis.

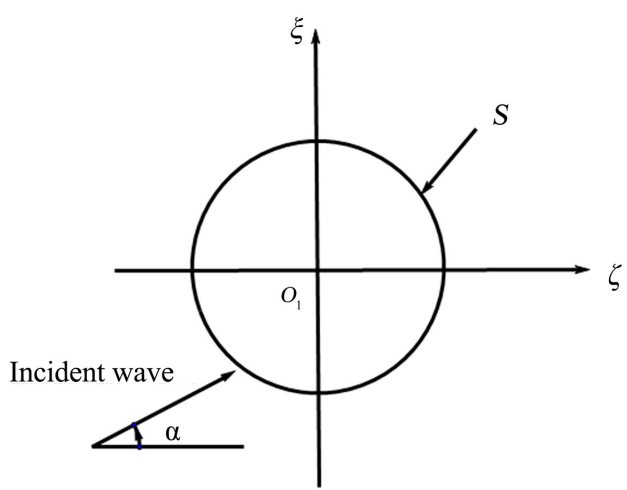

Figure 2. Image plane after conformal mapping.

$$
z=\omega(\eta)=c_{0} \eta+\sum_{n=1}^{\infty} c_{n} \frac{1}{\eta^{n}} .
$$

Substituting Equation (5) into Equations (2)-(4), we find

$$
\begin{gathered}
\phi^{\text {inc }}(\eta, \bar{\eta})=\phi_{0} \exp \left\{\frac{\mathrm{I} k_{p}}{2}\left[\omega(\eta) \mathrm{e}^{-\mathrm{I} \alpha}+\overline{\omega(\eta)} \mathrm{e}^{\mathrm{I} \alpha}\right]\right\}, \\
\phi^{\mathrm{ref}}(\eta, \bar{\eta})=\sum_{n=-\infty}^{\infty} A_{n} H_{n}^{(1)}\left(k_{p}|\omega(\eta)|\right)\left[\frac{\omega(\eta)}{|\omega(\eta)|}\right]^{n}, \\
\psi^{\mathrm{ref}}(\eta, \bar{\eta})=\sum_{n=-\infty}^{\infty} B_{n} H_{n}^{(1)}\left(k_{\mathrm{s}}|\omega(\eta)|\right)\left[\frac{\omega(\eta)}{|\omega(\eta)|}\right]^{n}
\end{gathered}
$$

Thus the total elastic field is described by $\phi=\phi^{\text {inc }}+\phi^{\text {ref }}$ and $\psi=\psi^{\text {ref }}$.

Meanwhile, the stress components $\sigma_{r r}, \sigma_{\theta \theta}$ and $\sigma_{r \theta}$ in $\eta$-plane are given by [24]

$$
\begin{aligned}
\sigma_{r r}= & -k_{p}^{2}(\lambda+\mu) \phi+\frac{2 \mu \eta^{2}}{r^{2} \overline{\omega^{\prime}(\eta)}} \frac{\partial}{\partial \eta}\left[\frac{1}{\omega^{\prime}(\eta)} \frac{\partial}{\partial \eta}(\phi+\mathrm{I} \psi)\right] \\
& +\frac{2 \mu \bar{\eta}^{2}}{r^{2} \omega^{\prime}(\eta)} \frac{\partial}{\partial \bar{\eta}}\left[\frac{1}{\overline{\omega^{\prime}(\eta)}} \frac{\partial}{\partial \bar{\eta}}(\phi-\mathrm{I} \psi)\right],
\end{aligned}
$$




$$
\begin{gathered}
\sigma_{\theta \theta}=-k_{p}^{2}(\lambda+\mu) \phi-\frac{2 \mu \eta^{2}}{r^{2} \overline{\omega^{\prime}(\eta)}} \frac{\partial}{\partial \eta}\left[\frac{1}{\omega^{\prime}(\eta)} \frac{\partial}{\partial \eta}(\phi+\mathrm{I} \psi)\right] \\
-\frac{2 \mu \bar{\eta}^{2}}{r^{2} \omega^{\prime}(\eta)} \frac{\partial}{\partial \bar{\eta}}\left[\frac{1}{\left.\overline{\omega^{\prime}(\eta)} \frac{\partial}{\partial \bar{\eta}}(\phi-\mathrm{I} \psi)\right],}\right. \\
\sigma_{r \theta}=\frac{2 \mathrm{I} \mu \eta^{2}}{r^{2} \overline{\omega^{\prime}(\eta)}} \frac{\partial}{\partial \eta}\left[\frac{1}{\omega^{\prime}(\eta)} \frac{\partial}{\partial \eta}(\phi+\mathrm{I} \psi)\right] \\
-\frac{2 \mathrm{I} \mu \bar{\eta}^{2}}{r^{2} \omega^{\prime}(\eta)} \frac{\partial}{\partial \bar{\eta}}\left[\frac{1}{\overline{\omega^{\prime}(\eta)}} \frac{\partial}{\partial \bar{\eta}}(\phi-\mathrm{I} \psi)\right] .
\end{gathered}
$$

Assume that the surface adheres perfectly to the bulk material without slipping. The equilibrium equations and the constitutive relations on the surface are expressed as

$$
\begin{gathered}
t_{\alpha}+\sigma_{\beta \alpha, \beta}^{s}=0, \\
\sigma_{i j} n_{i} n_{j}=\sigma_{\alpha \beta}^{s} \kappa_{\alpha \beta}, \\
\sigma_{\alpha \beta}^{s}=\tau_{0} \delta_{\alpha \beta}+2\left(\mu^{s}-\tau_{0}\right) \delta_{\alpha \gamma} \varepsilon_{\gamma \beta}+\left(\lambda^{s}+\tau_{0}\right) \varepsilon_{\gamma \gamma} \delta_{\alpha \beta} .
\end{gathered}
$$

where $n_{i}$ denotes the normal vector of the surface, $t_{\alpha}$ is the negative of the tangential component of the traction $t_{i}=\sigma_{i j} n_{j}$ in the $x_{\alpha}$ direction, and $\kappa_{\alpha \beta}$ is the curvature of the surface. Two dimensional indicators $\alpha, \beta$ value 1, 2 . The elastic property of anisotropic surface is characterized by two material constants $\mu^{s}$ and $\lambda^{s} . \sigma_{\alpha \beta}^{s}$ is surface stress tensor. $\tau_{0}$ is the residual surface tension under unstrained condition. Generally, the surface inertia force can be neglected in Equation (14) for the dynamic problems.

In the bulk, the classical elastic theory holds, and the isotropic constitutive relation reads as

$$
\sigma_{i j}=2 \mu\left(\varepsilon_{i j}+\frac{v}{1-2 v} \varepsilon_{k k} \delta_{i j}\right),
$$

For plane strain problems, the strain $\varepsilon_{\theta \theta}$ is given by

$$
\varepsilon_{\theta \theta}=\frac{1}{2 \mu}\left[(1-v) \sigma_{\theta \theta}-v \sigma_{r r}\right],
$$

Thus the surface stress $\sigma_{\theta \theta}^{s}$ is obtained as

$$
\sigma_{\theta \theta}^{s}=\tau_{0}+E^{s} \varepsilon_{\theta \theta} .
$$

with $E^{s}=2 \mu^{s}+\lambda^{s}-\tau_{0}$. Since the residual surface stress $\tau_{0}$ always induces an additional deformation field, independent of the external loading, we assume the residual surface stress $\tau_{0}$ to be zero in what follows.

The boundary conditions on the circular hole $(r=a)$ with surface effects are given from Equations (12) and (13) as

$$
\sigma_{r r}=\frac{\sigma_{\theta \theta}^{s}}{a}, \quad \sigma_{r \theta}=-\frac{\partial \sigma_{\theta \theta}^{s}}{a \partial \theta} .
$$

Thus in the $\eta$-plane, the boundary conditions on the unit circle with surface 
effects are given by

$$
\begin{aligned}
\sigma_{r r} & =\sigma_{\theta \theta}^{s}=\frac{E^{s}}{2 \mu}\left[(1-v) \sigma_{\theta \theta}-v \sigma_{r r}\right] \\
& =\frac{E^{s}}{2 \mu}\left\{(1-v)\left[-2 k_{p}(\lambda+\mu) \phi-\sigma_{r r}\right]-v \sigma_{r r}\right\} \\
& =-\frac{E^{s}}{2 \mu}\left[2(1-v) k_{p}^{2}(\lambda+\mu) \phi+\sigma_{r r}\right], \\
\sigma_{r \theta}= & -\frac{\partial \sigma_{\theta \theta}^{s}}{\partial \theta}=\frac{E^{s}}{2 \mu} \frac{\partial}{\partial \theta}\left[2(1-v) k_{p}^{2}(\lambda+\mu) \phi+\sigma_{r r}\right] .
\end{aligned}
$$

Substituting of Equations (6)-(8) and Equations (9)-(11) into Equations (19) and (20), we find

$$
\sum_{j=1}^{2}\left(\sum_{n=-\infty}^{\infty} E_{i j}^{n} X_{j}^{n}\right)=Y_{i}, i=1,2 .
$$

where $X_{1}^{n}=A_{n}, X_{2}^{n}=B_{n}$, and the elements of $E_{i j}^{n}$ are presented in Appen$\operatorname{dix}$ A.

Multiplying both side of Equation (21) by $\mathrm{e}^{-\mathrm{Il} \theta}$ and integrating between the interval $(-\pi, \pi)$, we have

$$
\sum_{j=1}^{2}\left(\sum_{n=-\infty}^{\infty} E_{i j l}^{n} X_{j}^{n}\right)=Y_{i l}, i=1,2 ; l=0, \pm 1, \cdots
$$

where $E_{i j l}^{n}=\frac{1}{2 \pi} \int_{-\pi}^{\pi} E_{i j}^{n} \mathrm{e}^{-1 l \theta} \mathrm{d} \theta, \quad Y_{i l}=\frac{1}{2 \pi} \int_{-\pi}^{\pi} Y_{i} \mathrm{e}^{-1 / \theta} \mathrm{d} \theta$.

We can get a set of infinite algebraic equations to determine the coefficients $A_{n}$ and $B_{n}$. After solving the linear equation system, the coefficients $A_{n}$ and $B_{n}$ can be obtained, then the elastic scattering fields due to the incident $\mathrm{P}$ wave can be determined.

\section{Numerical Results and Discussion}

The dynamic stress concentration factor (DSCF) is defined as the ratio between the hoop stress along the circumference of a cavity and the maximum amplitude of the incident stress wave at the same point

$$
\mathrm{DSCF}=\left|\frac{\sigma_{\theta \theta}}{\sigma_{0}}\right| .
$$

For compressive incident wave, the maximum amplitude of incident stress $\sigma_{0}$ is

$$
\sigma_{0}=-\mu k_{s}^{2} \phi_{0} .
$$

To illustrate the computational procedure, we consider the well-known case of a planar steady state compressive wave incident on a circular cavity. For a circular boundary, we take the mapping function as

$$
z=\omega(\eta)=a \eta
$$

Assume a harmonic plane $\mathrm{P}$ wave propagating in the $x$-axis positive direction. By truncating the infinite matrix in Equation (22) to $n=3$ and $l=3$. Let 
$s=E^{s} / 2 \mu a$. The Poisson ration is assumed to be $v=0.25$ for all calculation.

For low frequency incident wave with $k_{p} a=0.2$, surface parameter $s$ has significant effect on DSCF near a circle cavity. With the increase in parameter $s$, the DSCF continuously decrease around $\theta=\pi / 2$ but increases around $\theta=0$ and $\theta=\pi$ in Figure 3. Let $s=0$, the present nanosized circle cavity reduces to the classical cavity problem without a surface effect. These results are consistent with those in [13].

For simplicity, we assume that the incident planar compressive wave is coming along the direction of the $x$-axis. The distribution of dynamic stress concentration in the neighborhood of an elliptic cavity, with semi-major axis a and semi-minor axis $b$ can be made by choosing another mapping function

$$
z=\omega(\eta)=\frac{a+b}{2} \eta+\frac{a-b}{2} \bar{\eta} .
$$

Figure 4 shows distribution of the DSCF near the elliptic cavity for $a / b=3 / 2$ with the surface parameter, and the image is symmetric about $\theta=\pi / 2$. When $s \leq 0.5$, the DSCF is found the maximum value in $\theta=\pi / 2$. However, when $s=2$, the DSCF gets a minimum value. For the case of $s=0$, these results are consistent with those in [14].

Figure 5 illustrates distribution of the DSCF around the elliptic cavity with the different surface parameter for $a / b=2 / 3$. In this case, the image is symmetric about $\theta=\pi / 2$. With increasing surface parameter $s$, the DSCF declines continuously in $0.1 \pi<\theta<0.9 \pi$. When $s=0$, the DSCF almost unchanged around $\theta=\pi / 2$. But $s>0$, the DSCF dramatically increases around $\theta=\pi / 2$.

For a high frequency incident wave with $k_{p} a=\pi$, the dynamic stress concentration factor at the elliptical cavity surface is more complicated and the images appear multiple asymmetric peaks in Figure 6. For the different surface parameter $s$, distribution of the DSCF around the elliptical cavity surface is clearly depicted. Although the DSCF varies with the surface parameter is not very regular, the surface parameters take different values, the canyons of the image can be found at $\theta=0.1 \pi, \theta=0.5 \pi, \theta=0.9 \pi$.

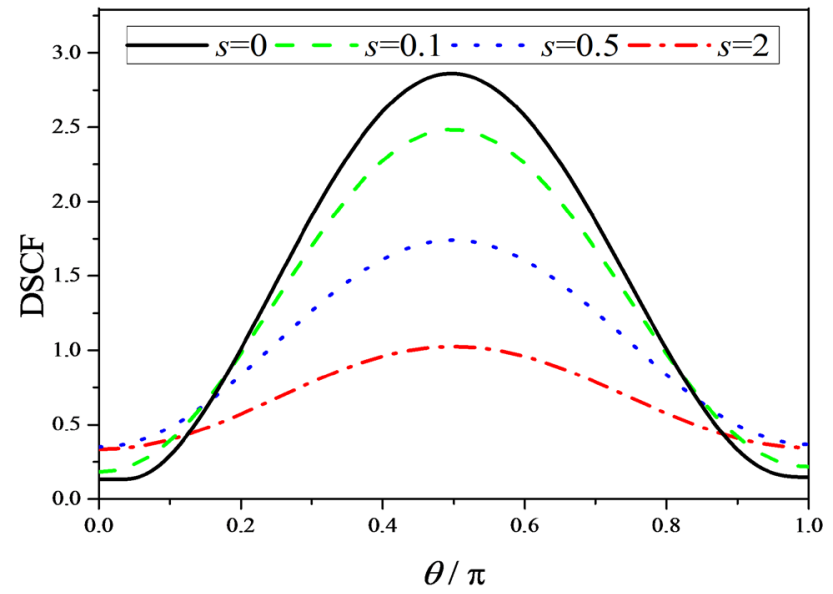

Figure 3. Effect of surface parameter $s$ on DSCF near a circle cavity for $k_{p} a=0.2$. 


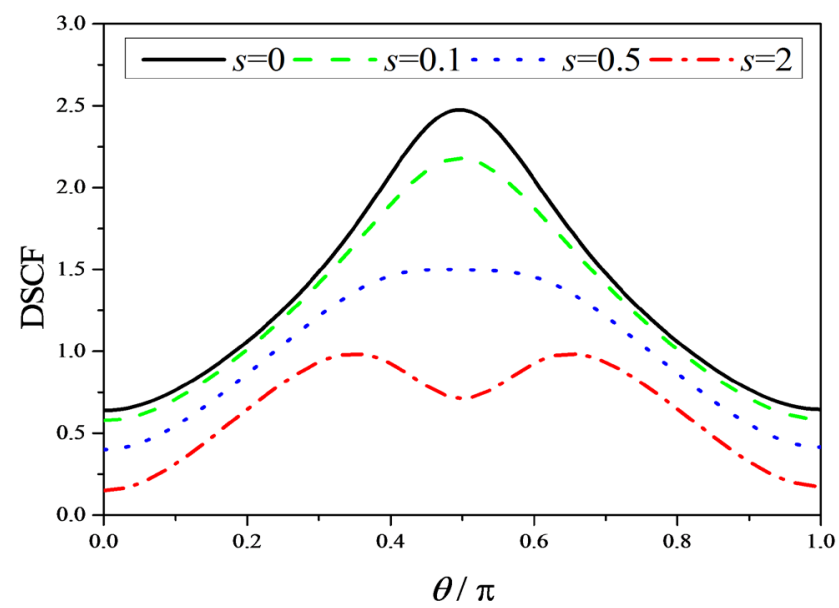

Figure 4. Effect of surface parameter $s$ on DSCF near an elliptic cavity for $k_{p} a=0.2, a / b=3 / 2$.

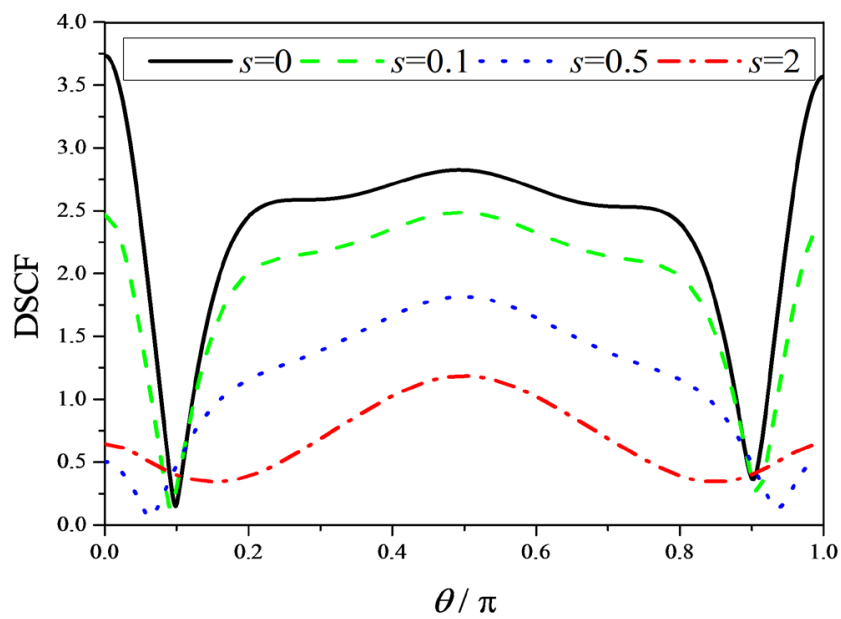

Figure 5. Effect of surface parameter $s$ on DSCF near an elliptic cavity for $k_{p} a=0.2, a / b=2 / 3$.

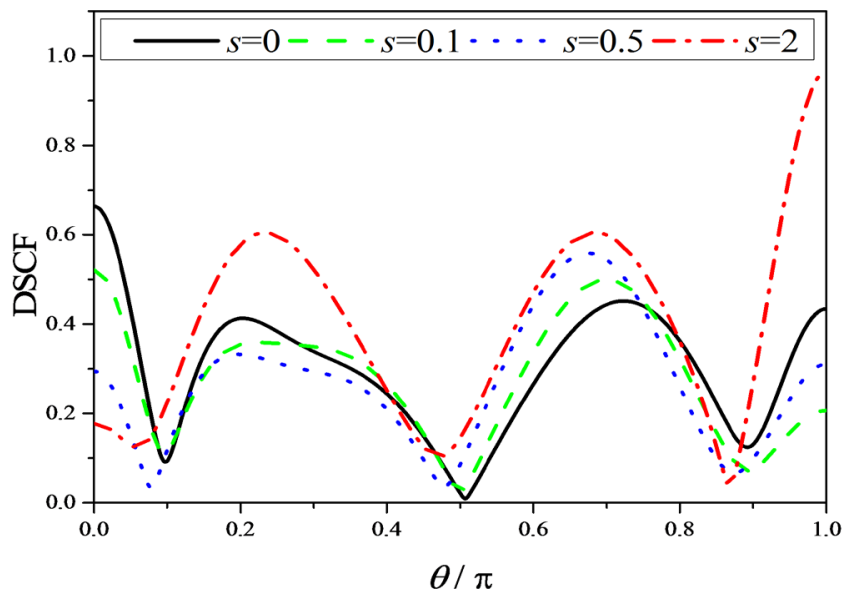

Figure 6. Effect of surface parameter $s$ on DSCF near an elliptic cavity for $k_{p} a=\pi, a / b=2 / 3$. 


\section{Conclusion}

In this paper, we have presented a method of complex functions to analyze the two dimensional problems of dynamic elasticity with non-circular geometry. The method is applied to evaluate the dynamic stress concentration factor around a circular and an elliptical cavity. Various type of scattering phenomena and dynamic stress concentrations due to a more general type of elastic inclusion can also be analyzed similarly.

\section{Acknowledgements}

The supports from the National Natural Science Foundation (Grant No. 11362009 and No. 11862014) are acknowledged.

\section{Conflicts of Interest}

The authors declare no conflicts of interest regarding the publication of this paper.

\section{References}

[1] Miller, R.E. and Shenoy, V.B. (2000) Size-Dependent Elastic Properties of Nanosized Structural Elements. Nanotechnology, 11, 139-145.

[2] Shenoy, V.B. (2002) Size-Dependent Rigidities of Nanosized Torsional Elements. International Journal of Solids and Structures, 39, 4039-4052. https://doi.org/10.1016/S0020-7683(02)00261-5

[3] Dingreville, R., Qu, J. and Cherkaoui, M. (2005) Surface Free Energy and Its Effect on the Elastic Behavior of Nano-Sized Particles, Wires and Films. Journal of the Mechanics and Physics of Solids, 53, 1827-1854. https://doi.org/10.1016/j.jmps.2005.02.012

[4] Duan, H.L., Wang, J., Huang, Z.P. and Karihaloo, B.L. (2005) Size-Dependent Effective Elastic Constants of Solids Containing Nano-Inhomogeneities with Interface Stress. Journal of the Mechanics and Physics of Solids, 53, 1574-1596. https://doi.org/10.1016/j.jmps.2005.02.009

[5] Chen, T., Dvorak, G.J. and Yu, C.C. (2007) Size-Dependent Elastic Properties of Unidirectional Nano-Composites with Interface Stresses. Acta Mechanica, 188, 39-54. https://doi.org/10.1007/s00707-006-0371-2

[6] Lei, D.X., Wang, L.Y. and Ou, Z.Y. (2012) Elastic Analysis for Nanocontact Problem with Surface Stress Effects under Shear Load. Journal of Nanomaterials, 6, 1-7. https://doi.org/10.1155/2012/505034

[7] Ou, Z.Y. and Lee, D.W. (2012) Effects of Interface Energy on Scattering of Plane Elastic Wave by a Nano-Sized Coated Fiber. Journal of Sound and Vibration, 331, 5623-5643. https://doi.org/10.1016/j.jsv.2012.07.023

[8] Ou, Z.Y. and Lee, D.W. (2012) Effects of Interface Energy on Multiple Scattering of Plane Compressional Waves by Two Cylindrical Fibers. International Journal of Applied Mechanics, 4, 401-419. https://doi.org/10.1142/S1758825112500408

[9] Zhang, Q.F., Wang, G.F. and Schiavone, P. (2011) Diffraction of Plane Compressional Waves by an Array of Nanosized Cylindrical Holes. Journal of Applied Mechanics-Transactions of the ASME, 78, 856-875. https://doi.org/10.1115/1.4002529

[10] Ru, Y. (2015) Surface Effect on Diffractions of Elastic Waves and Stress Concentra- 
tion Near a Cluster of Cylindrical Nanoholes Arranged as Quadrate Shape. Advances in Materials Science and Engineering, 5, 1-8. https://doi.org/10.1155/2015/134975

[11] Ou, Z.Y., Liu, C. and Liu, X.W. (2013) Effects of Surface Elasticity on Scattering of P Waves by an Elastic Half Plane with a Nanosized Semi-Cylindrical Inclusion. Applied Mechanics and Materials, 303, 2661-2666. https://doi.org/10.4028/www.scientific.net/AMM.303-306.2661

[12] Wang, G.F. and Wang, T.J. (2006) Deformation around a Nanosized Elliptical Hole with Surface Effect. Applied Physics Letters, 89, 1901-1916. https://doi.org/10.1063/1.2362988

[13] Wang, G.F., Wang, T.J. and Feng, X.Q. (2006) Surface Effects on the Diffraction of Plane Compressional Waves by a Nanosized Circular Hole. Journal of Applied Physics, 89, 1923-1925. https://doi.org/10.1063/1.2403899

[14] Liu, D.K., Gai, B.Z. and Tao, G.Y. (1982) Applications of the Method of Complex Functions to Dynamic Stress Concentrations. Wave Motion, 4, 293-304. https://doi.org/10.1016/0165-2125(82)90025-7

[15] Ou, Z.Y. and Zhao, C.H. (2018) Stress Concentration around a Nanosized Arbitrarily Shaped Hole in Elastic Half-Space under Uniformly Distributed Load. Journal of Lanzhou University of Technology, 44, 160-165.

[16] Sommerfeld, A. (2004) Mathematical Theory of Diffraction. Springer Science Business Media. Springer Nature Switzerland AG, Basel, Switzerland. https://doi.org/10.1007/978-0-8176-8196-8

[17] Lee, V.W. and Karl, J. (1992) Diffraction of SV Waves by Underground, Circular, Cylindrical Cavities. Soil Dynamics and Earthquake Engineering, 11, 445-456. https://doi.org/10.1016/0267-7261(92)90008-2

[18] Gilbert, F. and Knopoff, L. (1959) Scattering of Impulsive Elastic Waves by a Rigid Cylinder. Journal of the Acoustical Society of America, 31, 1169-1175. https://doi.org/10.1121/1.1907846

[19] Mow, C.C. and Pao, Y.H. (1973) Diffraction of Elastic Waves and Dynamic Stress Concentrations. Journal of Applied Mechanics, 40, 872-1500.

[20] Wang, J.H., Lu, J.F. and Zhou, X.L. (2009) Complex Variable Function Method for the Scattering of Plane Waves by an Arbitrary Hole in a Porous Medium. European Journal of Mechanica-A/Solids, 28, 582-590. https://doi.org/10.1016/j.euromechsol.2008.09.005

[21] Zitron, N.R. (1967) Multiple Scattering of Elastic Waves by Two Arbitrary Cylinders. Journal of the Acoustical Society of America, 42, 620-624. https://doi.org/10.1121/1.1910633

[22] Liu, D.K. and Chen, Z.G. (2004) Scattering of SH-Wave by Cracks Originating at an Elliptic Hole and Dynamic Stress Intensity Factor. Applied Mathematics and Mechanics, 25, 1047-1056. https://doi.org/10.1007/BF02438354

[23] Fang, X.Q. and Jin, H.X. (2016) Visco-Elastic Imperfect Bonding Effect on Dynamic Response of a Non-Circular Lined Tunnel Subjected to P and SV Waves. Soil Dynamics and Earthquake Engineering, 88, 1-7. https://doi.org/10.1016/j.soildyn.2016.05.014

[24] Liu, Q.J., Zhao, M.J. and Wang, L.H. (2013) Scattering of Plane P, SV or Rayleigh Waves by a Shallow Lined Tunnel in an Elastic Half Space. Soil Dynamics and Earthquake Engineering, 49, 52-63. https://doi.org/10.1016/j.soildyn.2013.02.007 


\section{Appendix A}

$$
\begin{aligned}
& E_{11}^{n}=\left(1+\frac{E^{s}}{2 \mu}\right)\left\{-k_{p}^{2}(\lambda+\mu) H_{n}^{(1)}\left(k_{p}|\omega(\eta)|\right)\left[\frac{\omega(\eta)}{|\omega(\eta)|}\right]^{n}\right. \\
& +\frac{\mu k_{p}^{2} \eta^{2} \omega^{\prime}(\eta)}{2 \overline{\omega^{\prime}(\eta)}} H_{n-2}^{(1)}\left(k_{p}|\omega(\eta)|\right)\left[\frac{\omega(\eta)}{|\omega(\eta)|}\right]^{n-2} \\
& \left.+\frac{\mu k_{p}^{2} \bar{\eta}^{2} \overline{\omega^{\prime}(\eta)}}{2 \omega^{\prime}(\eta)} H_{n+2}^{(1)}\left(k_{p}|\omega(\eta)|\right)\left[\frac{\omega(\eta)}{|\omega(\eta)|}\right]^{n+2}\right\} \\
& +\frac{E^{s}}{\mu}(1-v) k_{p}^{2}(\lambda+\mu) H_{n}^{(1)}\left(k_{p}|\omega(\eta)|\right)\left[\frac{\omega(\eta)}{|\omega(\eta)|}\right]^{n} \\
& E_{12}^{n}=\left(1+\frac{E^{s}}{2 \mu}\right)\left\{\frac{\mathrm{I} \mu k_{s}^{2} \eta^{2} \omega^{\prime}(\eta)}{2 \overline{\omega^{\prime}(\eta)}} H_{n-2}^{(1)}\left(k_{s}|\omega(\eta)|\right)\left[\frac{\omega(\eta)}{|\omega(\eta)|}\right]^{n-2}\right. \\
& \left.-\frac{\mathrm{I} \mu k_{s}^{2} \bar{\eta}^{2} \overline{\omega^{\prime}(\eta)}}{2 \omega^{\prime}(\eta)} H_{n+2}^{(1)}\left(k_{s}|\omega(\eta)|\right)\left[\frac{\omega(\eta)}{|\omega(\eta)|}\right]^{n+2}\right\} \\
& Y_{1}=\left\{\left(1+\frac{E^{s}}{2 \mu}\right)\left[\lambda+\mu+\frac{\mu \eta^{2} \omega^{\prime}(\eta)}{2 \overline{\omega^{\prime}(\eta)}} \mathrm{e}^{-2 \mathrm{I} \alpha}+\frac{\mu \bar{\eta}^{2} \overline{\omega^{\prime}(\eta)}}{2 \omega^{\prime}(\eta)} \mathrm{e}^{2 \mathrm{I} \alpha}\right]\right. \\
& \left.-\frac{E^{s}}{\mu}(\lambda+\mu)(1-v)\right\} k_{p}^{2} \phi_{0} \exp \left\{\frac{\mathrm{I} k_{p}}{2}\left[\omega(\eta) \mathrm{e}^{-\mathrm{I} \alpha}+\overline{\omega(\eta)} \mathrm{e}^{\mathrm{I} \alpha}\right]\right\} \\
& E_{21}^{n}=\frac{\mathrm{I} \mu k_{p}^{2} \eta^{2} \omega^{\prime}(\eta)}{2 \overline{\omega^{\prime}(\eta)}} H_{n-2}^{(1)}\left(k_{p}|\omega(\eta)|\right)\left[\frac{\omega(\eta)}{|\omega(\eta)|}\right]^{n-2} \\
& -\frac{\mathrm{I} \mu k_{p}^{2} \bar{\eta}^{2} \overline{\omega^{\prime}(\eta)}}{2 \omega^{\prime}(\eta)} H_{n+2}^{(1)}\left(k_{p}|\omega(\eta)|\right)\left[\frac{\omega(\eta)}{|\omega(\eta)|}\right]^{n+2}+\frac{E^{s}}{2 \mu} \frac{\partial}{\partial \theta} F_{1} \\
& E_{22}^{n}=-\frac{\mu k_{s}^{2} \eta^{2} \omega^{\prime}(\eta)}{2 \overline{\omega^{\prime}(\eta)}} H_{n-2}^{(1)}\left(k_{s}|\omega(\eta)|\right)\left[\frac{\omega(\eta)}{|\omega(\eta)|}\right]^{n-2} \\
& -\frac{\mu k_{s}^{2} \bar{\eta}^{2} \overline{\omega^{\prime}(\eta)}}{2 \omega^{\prime}(\eta)} H_{n+2}^{(1)}\left(k_{s}|\omega(\eta)|\right)\left[\frac{\omega(\eta)}{|\omega(\eta)|}\right]^{n+2}+\frac{E^{s}}{2 \mu} \frac{\partial}{\partial \theta} F_{2} \\
& Y_{2}=\left[\frac{\mathrm{I} \mu \eta^{2} \omega^{\prime}(\eta)}{2 \overline{\omega^{\prime}(\eta)}} \mathrm{e}^{-2 \mathrm{I} \alpha}-\frac{\mathrm{I} \mu \bar{\eta}^{2} \overline{\omega^{\prime}(\eta)}}{2 \omega^{\prime}(\eta)} \mathrm{e}^{2 \mathrm{I} \alpha}\right] k_{p}^{2} \phi_{0} \\
& \times \exp \left\{\frac{\mathrm{I} k_{p}}{2}\left[\omega(\eta) \mathrm{e}^{-\mathrm{I} \alpha}+\overline{\omega(\eta)} \mathrm{e}^{\mathrm{I} \alpha}\right\}-\frac{E^{s}}{2 \mu} \frac{\partial}{\partial \theta} F_{3}\right. \\
& F_{1}=(2 v-1) k_{p}^{2}(\lambda+\mu) H_{n}^{(1)}\left(k_{p}|\omega(\eta)|\right)\left[\frac{\omega(\eta)}{|\omega(\eta)|}\right]^{n} \\
& -\frac{\mu k_{p}^{2} \eta^{2} \omega^{\prime}(\eta)}{2 \overline{\omega^{\prime}(\eta)}} H_{n-2}^{(1)}\left(k_{p}|\omega(\eta)|\right)\left[\frac{\omega(\eta)}{|\omega(\eta)|}\right]^{n-2}
\end{aligned}
$$




$$
\begin{gathered}
-\frac{\mu k_{p}^{2} \bar{\eta}^{2} \overline{\omega^{\prime}(\eta)}}{2 \omega^{\prime}(\eta)} H_{n+2}^{(1)}\left(k_{p}|\omega(\eta)|\right)\left[\frac{\omega(\eta)}{|\omega(\eta)|}\right]^{n+2} \\
F_{2}=-\frac{\mathrm{I} \mu k_{s}^{2} \eta^{2} \omega^{\prime}(\eta)}{2 \overline{\omega^{\prime}(\eta)}} H_{n-2}^{(1)}\left(k_{s}|\omega(\eta)|\right)\left[\frac{\omega(\eta)}{|\omega(\eta)|}\right]^{n-2} \\
+\frac{\mathrm{I} \mu k_{s}^{2} \bar{\eta}^{2} \overline{\omega^{\prime}(\eta)}}{2 \omega^{\prime}(\eta)} H_{n+2}^{(1)}\left(k_{s}|\omega(\eta)|\right)\left[\frac{\omega(\eta)}{|\omega(\eta)|}\right]^{n+2} \\
F_{3}=\left[(2 v-1)(\lambda+\mu)+\frac{\mu \eta^{2} \omega^{\prime}(\eta)}{2 \overline{\omega^{\prime}(\eta)}} \mathrm{e}^{-2 \mathrm{I} \alpha}+\frac{\mu \bar{\eta}^{2} \overline{\omega^{\prime}(\eta)}}{2 \omega^{\prime}(\eta)} \mathrm{e}^{2 \mathrm{I} \alpha}\right] k_{p}^{2} \phi_{0} \\
\quad \times \exp \left\{\frac{\mathrm{I} k_{p}}{2}\left[\omega(\eta) \mathrm{e}^{-\mathrm{I} \alpha}+\overline{\omega(\eta)} \mathrm{e}^{\mathrm{I} \alpha}\right]\right\}
\end{gathered}
$$

